\title{
Modélisation de la surface piézométrique des aquifères fissurés en zone de socle précambrien métamorphisé : cas du bassin versant du Sassandra (sud-ouest de la Côte d'Ivoire) Piezometric modeling in precambrian fractured metamorphosed hard-rock: case of the Sassandra watershed (South-Western Côte d'Ivoire)
}

\author{
Théodore Koffi Yao, Olivier Fouché, Emmanuel Konan Kouadio, Marie-Solange \\ Oga et Théophile Lasm
}

Volume 28, numéro 2, 2015

Reçu le 29 juillet 2013, accepté le 18 août 2014

URI : https://id.erudit.org/iderudit/1032293ar

DOI : https://doi.org/10.7202/1032293ar

Aller au sommaire du numéro

Éditeur(s)

Université du Québec - INRS-Eau, Terre et Environnement (INRS-ETE)

ISSN

1718-8598 (numérique)

Découvrir la revue

Citer cet article

Yao, T. K., Fouché, O., Kouadio, E. K., Oga, M.-S. \& Lasm, T. (2015). Modélisation de la surface piézométrique des aquifères fissurés en zone de socle précambrien métamorphisé : cas du bassin versant du Sassandra (sud-ouest de la Côte d'Ivoire). Revue des sciences de l'eau / Journal of Water Science, 28(2), 105-117. https://doi.org/10.7202/1032293ar
Résumé de l'article

Les niveaux piézométriques dans les aquifères de socle en Côte d'Ivoire sont mal connus du fait de la complexité du substratum géologique. Ce travail a pour objectif de modéliser l'altitude du niveau piézométrique des aquifères situés en milieu cristallin et cristallophyllien, notamment dans le bassin versant du Sassandra, au sud-ouest de la Côte d'Ivoire. En exploitant la méthode de la corrélation linéaire entre la dénivellation de la surface du sol et la surface de base des thalwegs d'une part et la dénivellation entre la surface piézométrique et la surface de base des thalwegs d'autre part, nous avons établi des équations qui permettent de déterminer les niveaux piézométriques et de compenser ainsi les lacunes dans leur connaissance. Ces travaux s'appuient sur des données de terrain, des fiches techniques de forage, des cartes (topographique et géologique) et un extrait du Modèle numérique d'Altitude (MNA) du sud-ouest ivoirien.

L'interprétation mathématique des résultats a permis, au travers des équations établies, de calculer directement le niveau piézométrique en tout point de la zone d'étude. La carte piézométrique ainsi obtenue montre que le niveau piézométrique est en moyenne $7 \mathrm{~m}$ en dessous du sol lorsque l'on prend comme référence la surface de base des thalwegs. La surface piézométrique est corrélée à la surface topographique. La superposition de la carte piézométrique à celle du réseau hydrographique montre qu'à l'échelle de l'analyse réalisée, la circulation de l'eau souterraine est calquée sur celle des eaux de surface ; bassins versants hydrogéologique et topographique se superposent, montrant ainsi le lien entre les eaux de surface et les eaux souterraines. Ce résultat pourra aider à la gestion intégrée des ressources en eau souterraine et de surface surtout face à la vulnérabilité aux pesticides qui sont fréquemment utilisés dans la zone étudiée. 
MODÉLISATION DE LA SURFACE PIÉZOMÉTRIQUE DES AQUIFÈRES FISSURÉS EN ZONE DE SOCLE PRÉCAMBRIEN MÉTAMORPHISÉ : CAS DU BASSIN VERSANT DU SASSANDRA (SUD-OUEST DE LA CÔTE D'IVOIRE)

\author{
Piezometric modeling in precambrian fractured metamorphosed hard-rock: case of the Sassandra watershed \\ (South-Western Côte d'Ivoire)
}

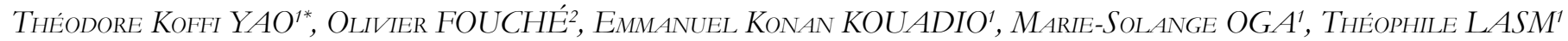

${ }^{1}$ Université Félix Houphouët-Boigny, Abidjan, UFR-STRM, 22 BP 582 Abidjan 22, Côte d'Ivoire

${ }^{2}$ Conservatoire national des arts et métiers de Paris, Dépt ICENER - Service de Géotechnique, 2 Rue Conté, 75003 Paris, France

Reçu le 29 juillet 2013, accepté le 18 août 2014

\section{RÉSUMÉ}

Les niveaux piézométriques dans les aquifères de socle en Côte d'Ivoire sont mal connus du fait de la complexité du substratum géologique. Ce travail a pour objectif de modéliser l'altitude du niveau piézométrique des aquiferes situés en milieu cristallin et cristallophyllien, notamment dans le bassin versant du Sassandra, au sud-ouest de la Côte d'Ivoire. En exploitant la méthode de la corrélation linéaire entre la dénivellation de la surface du sol et la surface de base des thalwegs d'une part et la dénivellation entre la surface piézométrique et la surface de base des thalwegs d'autre part, nous avons établi des équations qui permettent de déterminer les niveaux piézométriques et de compenser ainsi les lacunes dans leur connaissance. Ces travaux s'appuient sur des données de terrain, des fiches techniques de forage, des cartes (topographique et géologique) et un extrait du Modèle numérique d'Altitude (MNA) du sudouest ivoirien.

L'interprétation mathématique des résultats a permis, au travers des équations établies, de calculer directement le niveau piézométrique en tout point de la zone d'étude. La carte piézométrique ainsi obtenue montre que le niveau piézométrique est en moyenne $7 \mathrm{~m}$ en dessous du sol lorsque l'on prend comme référence la surface de base des thalwegs. La surface piézométrique est corrélée à la surface topographique. La superposition de la carte piézométrique à celle du réseau 
hydrographique montre qu'à l'échelle de l'analyse réalisée, la circulation de l'eau souterraine est calquée sur celle des eaux de surface ; bassins versants hydrogéologique et topographique se superposent, montrant ainsi le lien entre les eaux de surface et les eaux souterraines. Ce résultat pourra aider à la gestion intégrée des ressources en eau souterraine et de surface surtout face à la vulnérabilité aux pesticides qui sont fréquemment utilisés dans la zone étudiée.

Mots clés : Aquifere de socle, eau souterraine, surface piézométrique, modèle numérique d'altitude, thalwegs, modélisation.

\section{ABSTRACT}

The piezometric levels in hard-rock from Côte d'Ivoire are poorly understood because of the complexity of the geological substratum. This work aims to model the piezometric level of aquifers located in crystalline and crystallophyllian basement, particularly in the Sassandra Watershed (south-western Côte d'Ivoire). By exploiting, on the one hand the method of linear correlation between the elevation of ground surface and base surface of thalwegs, and on the other hand the difference in elevation between the piezometric surface and the base surface thalwegs, we have established equations to determine groundwater levels and thereby compensate gaps in our knowledge. This study is based on field data, drilling datasheet, maps (topographic and geological) and an extract of the Digital Elevation Model (DEM) in south-western Cote d'Ivoire.

The mathematical interpretation of the results, using established equations, helped us to calculate directly the piezometric level at any point in the study area. The piezometric map obtained shows that groundwater level is on average $7 \mathrm{~m}$ below ground when we take as reference the base surface of the thalwegs. The piezometric surface is correlated with the topographic surface. On the scale of the analysis carried out, the superposition of the piezometric map on the hydrographic network shows that flow of groundwater is modeled on that of the surface water. This observation supports the idea that hydrogeological and topographical watersheds overlap. This is logical considering the low permeability of hard-rock media and the relative homogeneity of their hydrodynamic properties on the scale of this approach. This result may help the integrated management of groundwater and surface water resources, especially regarding their vulnerability to pesticides, which are frequently used in the study area.

\section{Keywords: Hard-rock aquifers, groundwater, piezometric level, digital elevation model, thalwegs, modeling}

\section{INTRODUCTION}

La gestion des ressources en eau implique la prise en compte conjointe des eaux de surface et des nappes. Ces dernières interviennent de façon non négligeable dans l'alimentation des rivières, le transfert des éléments polluants et l'alimentation en eau des êtres vivants (KAMAGATÉ, 2006 ; LAROCQUE et PHARAND, 2010).

Les ressources en eau souterraine de socle en Côte d'Ivoire sont mal connues (BIÉMI, 1992). Elles sont situées dans un contexte hydrogéologique complexe (roches cristallines d'origine plutonique et métamorphique) où l'évaluation de la réserve en eau disponible reste difficile compte tenu des technologies et des méthodes qui sont mises en œuvre jusqu’à présent. L'étude hydrodynamique et la disponibilité de l'eau souterraine nécessitent la connaissance du niveau piézométrique des nappes (ENGALENC, 1978). Dans le Sud-Ouest, à l'instar de plusieurs régions de socle en Côte d'Ivoire, il n'existe pas de données synchrones sur la piézométrie des nappes.

Les premières études portant sur les variations piézométriques des nappes remontent à celles de BRGM-CIEH (1972), JOSEPH (1969), LELONG et LEMOINE (1968) dans les arènes granitiques et de BELIARD et al. (1973) dans les altérites de schistes. Une carte piézométrique de l'ancienne "boucle du cacao " (centre-est) de la Côte d'Ivoire avec les niveaux piézométriques mesurés dans des puits peu profonds a été dressée (LENCK, 1977). Une étude piézométrique complète a été réalisée en ce qui concerne l'horizon fissuré plus profond par FAILLAT et LEBLOND (1982) au niveau d'un site expérimental à Yamoussoukro (Centre de la Côte d'Ivoire).

Aucune étude piézométrique n’a été réalisée auparavant sur le bassin versant du Sassandra. En dehors des rapports de forages issus des campagnes d'hydraulique villageoise qui donnent une idée approximative du niveau de la nappe, les données exploitables sont quasi inexistantes. La modélisation permet de compenser le déficit de données par la mise en place d'équations qui permettent de déterminer l'altitude du niveau piézométrique. Cette modélisation a été possible en prenant en compte le caractère très fracturé du socle du bassin versant du Sassandra dans le département de Soubré (YAO, 2009).

\section{MATÉRIEL ET MÉTHODES}

\subsection{Zone d'étude}

\subsubsection{Caractéristiques géographiques et climatiques}

La zone d'étude se localise sur le sud du bassin versant $\mathrm{du}$ Sassandra et couvre une superficie de $6000 \mathrm{~km}^{2}$. Elle est située dans le département de Soubré, au Sud-Ouest de la 
Côte d'Ivoire, entre les latitudes 530'07 et 600'19 Nord et les longitudes $6^{\circ} 19^{\prime} 37$ et $6^{\circ} 53^{\prime} 04$ Ouest (Figure 1). Le climat est de type subéquatorial, caractérisé par une pluviométrie abondante (entre $1600 \mathrm{~mm}$ et $1800 \mathrm{~mm}$ par an). La température oscille entre 29 et $32^{\circ} \mathrm{C}$.

$\mathrm{Au}$ plan géomorphologique, la région est une vaste pénéplaine légèrement ondulée, d'altitude moyenne de $200 \mathrm{~m}$ avec une pente de $2 \%$ vers la mer (YAO, 2009). Elle est constituée de plaines et de bas plateaux occupés par la forêt sempervirente, la forêt semi-décidue et de vastes plantations de produits d'exportation (cacao, café, palmier à huile et hévéa). L'exploitation de ces plantations nécessite une main d'œuvre importante qui engendre du coup une pression croissante sur les ressources en eau.

Le département de Soubré où se situe la zone étudiée est couvert à plus de $90 \%$ par le bassin du fleuve Sassandra qui comprend ses deux principaux affluents (Lobo et Davo) et de nombreuses rivières. En outre, un grand nombre de sources et cours d'eau s'y trouvent, ainsi que le grand lac artificiel de Buyo (au nord-ouest du département). D’une capacité de
$8,3 \cdot 10^{9} \cdot \mathrm{m}^{3}$, il résulte de la création du barrage hydroélectrique de Buyo en 1980 (ARDOUIN, 2004).

\subsubsection{Contexte géologique et hydrogéologique}

Les principales formations géologiques du département de Soubré appartiennent au domaine éburnéen (2 500 à $1550 \mathrm{Ma}$ ) et sont caractérisées par une grande diversité de massifs plutoniques intrusifs dans un encaissant métamorphique (gneiss, migmatites) plus ancien (Mégacycle Libérien). Dans le compartiment nord-ouest de la région, on observe des roches flyschoïdes constituées de divers schistes et de grès, produits du démantèlement de la chaîne libérienne (Figure 2).

Plusieurs phases de déformation ont affecté cet ensemble géologique et ont abouti à la mise en place d'une fracturation intense (TAGINI, 1971). Les fractures s'orientent majoritairement suivant trois directions principales : SSWNNE, SW-NE et N-S avec plus de $69 \%$ qui ont des longueurs comprises entre $0,3 \mathrm{~km}$ et $6 \mathrm{~km}$ (YAO et al., 2012). Quelques failles de longueur maximale $15 \mathrm{~km}$, orientées NW-SE ou parfois N-S sont marquées par des mylonites (PAPON et LEMARCHAND, 1973). L'accident tectonique le plus remarquable est la faille de Hana-Lobo (NE-SW) (Figure 2). Elle

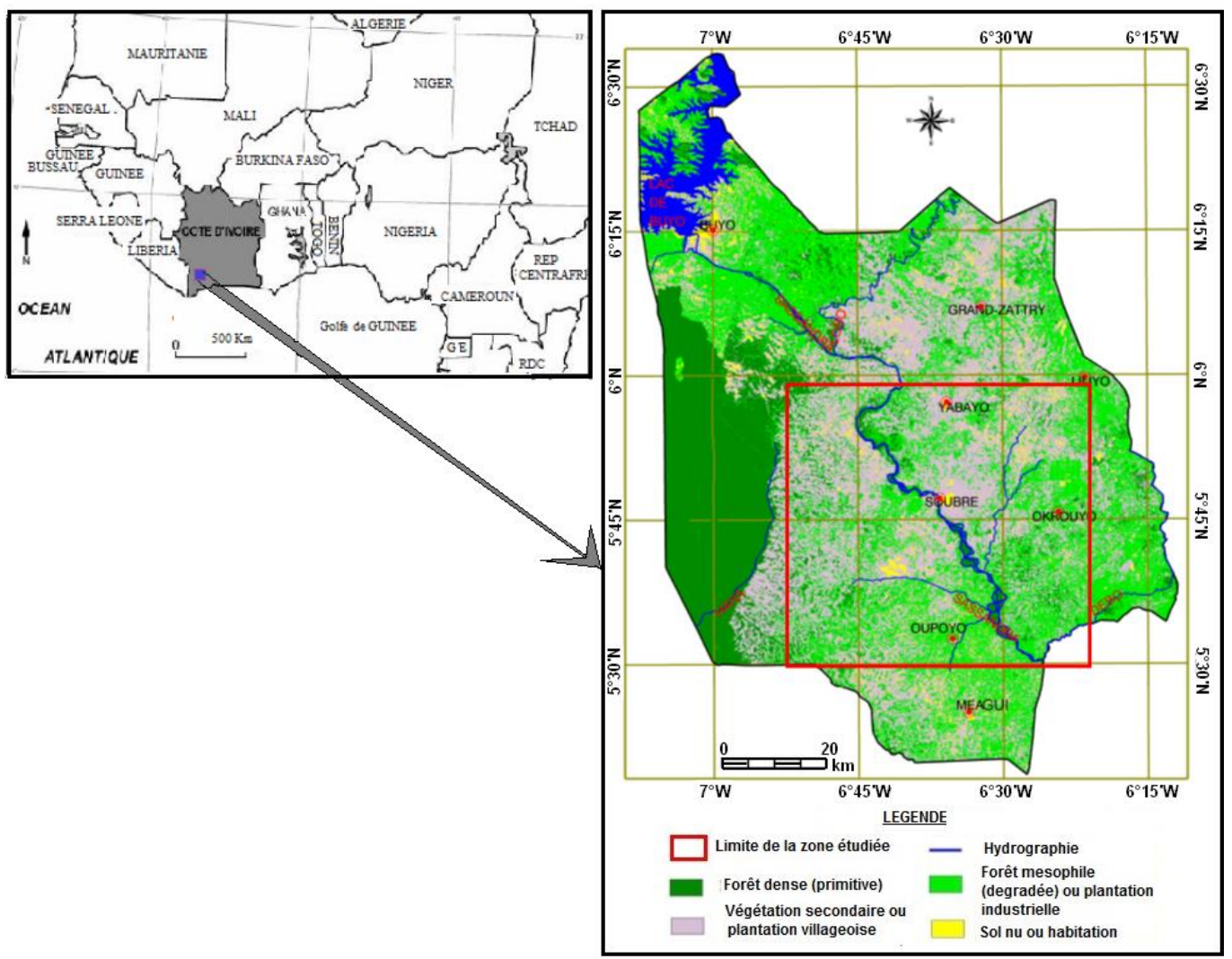

Figure 1. Localisation de la zone d'étude. Study area location. 

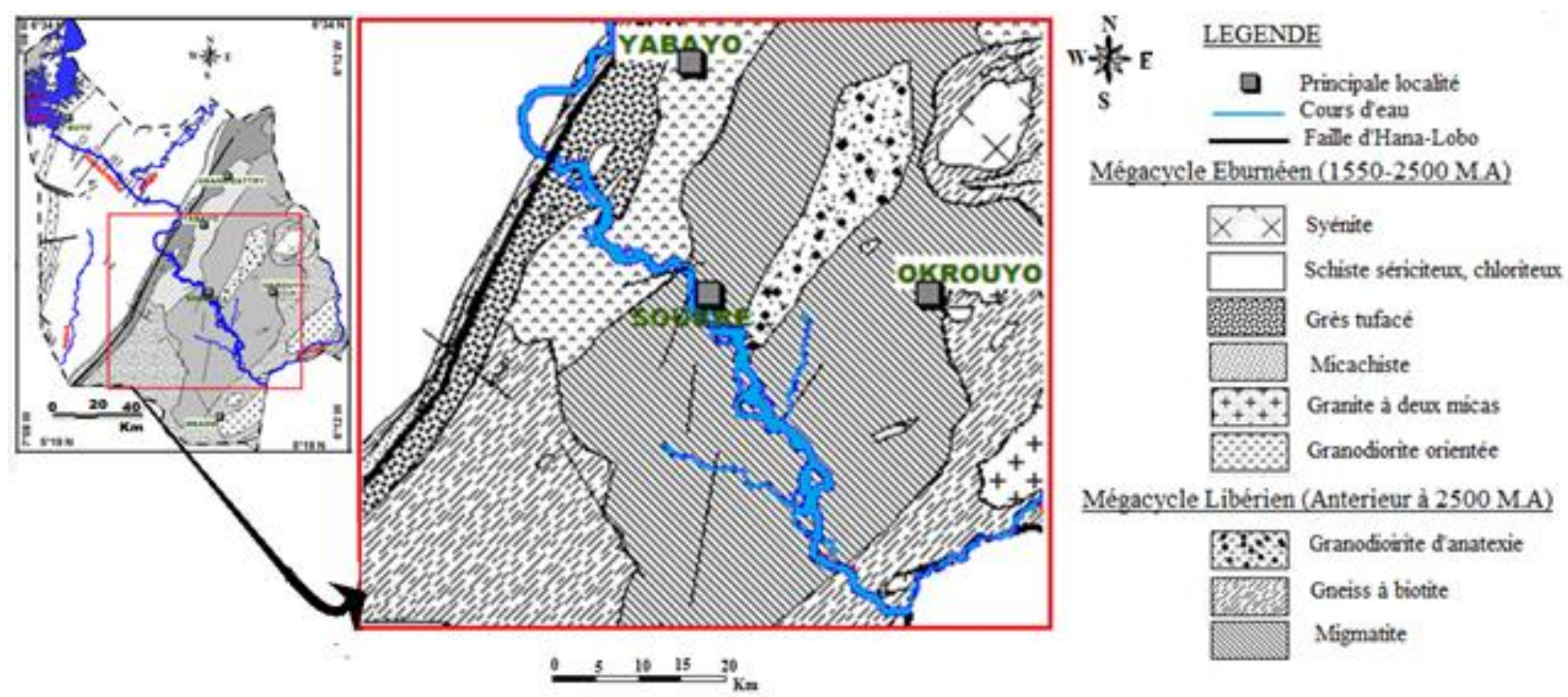

Figure 2. Carte géologique simplifiée de la zone d'étude (carte modifiée d'après PAPON et LEMARCHAND, 1973).

Simplified geological map of the study area (modified from PAPON and LEMARCHAND, 1973).

traverse la partie nord-ouest du cadre d'étude sur une distance d'environ $45 \mathrm{~km}$. Elle limite la zone des formations flyschoïdes des roches plutoniques et leur encaissant métamorphique.

Dans ce contexte géologique, les eaux souterraines circulent dans trois types de réservoirs qui se superposent (DEWANDEL et al., 2011 ; LACHASSAGNE et al., 2011 ; WYNS et al., 1999, 2004). En surface se localise le réservoir d'altérites, argilosableux, d'épaisseur variable (10 à $40 \mathrm{~m}$ ) et de faible perméabilité $\left(10^{-6}\right.$ à $\left.10^{-5} \cdot \mathrm{m} \cdot \mathrm{s}^{-1}\right)$, avec un coefficient d'emmagasinement de l'ordre de $10^{-2}$ à $10^{-1}$ et une transmissivité de $10^{-5}$ à $10^{-4} \cdot \mathrm{m}^{2} \cdot \mathrm{s}^{-1}$ (FAILLAT et BLAVOUX, 1989). Le réservoir de fissures est situé juste en-dessous du réservoir d'altérites et est beaucoup plus perméable. Ces deux premiers réservoirs fonctionnent comme un aquifère composite (DEWANDEL et al., 2006 ; LACHASSAGNE et al., 2011) sous lequel se trouvent parfois d'autres types de discontinuités (filons de quartz, d'aplites ou de pegmatites par exemple, diaclases, failles, contacts lithologiques...), localement aquiferres (DEWANDEL et al., 2011).

\subsection{Données}

La base de données initiale est composée d'un modèle numérique d'altitude (MNA), de cartes topographique et géologique, de données hydroclimatiques, de données techniques de forages et des coordonnées géographiques de certains forages.
Des informations pas toujours exhaustives ont été relevées dans 220 dossiers de forages recueillis auprès de la SousDirection Territoriale de l'Hydraulique de San Pedro. Les informations concernent le niveau piézométrique (mesure piézométrique ponctuelle), la profondeur du forage, sa cote topographique (altitude par rapport à la mer) et ses coordonnées géographiques. Les deux dernières informations ont été complétées par des relevés sur le terrain à l'aide d'un GPS GARMIN 76.

Le MNA de la région a été obtenu à partir des images de Shuttle Radar Topographic Mission (SRTM) réalisée en février 2000 depuis la navette spatiale Endeavour. Il fournit des valeurs d'altitude qui reposent sur un quadrillage de $0,324 \mathrm{~s}$ d'arc en coordonnées géographiques. L'incertitude absolue en $\mathrm{Z}$ est d'environ $10 \mathrm{~m}$ et son incertitude relative est de $4 \mathrm{~m}$ (MRNF, 2011).

\subsection{Méthodes}

Les données ponctuelles sur le niveau piézométrique disponibles n'étant pas assez nombreuses pour étudier la piézométrie, nous avons eu recours à la "modélisation ». La méthode mise en ouvre pour évaluer la cote de la surface piézométrique en tous lieux dans un contexte de socle altéré a été développée et validée par WYNS et al. (2004). Le principe de cette méthode est illustré par la figure 3. Elle est fondée sur plusieurs hypothèses : 
- on considère que les cours d'eau permanents constituent les exutoires des eaux souterraines ;

- les niveaux piézométriques des ouvrages situés à proximité d'un cours d'eau pérenne sont toujours au-dessus du niveau de ces cours d'eau ;

- en phase de tarissement, ces niveaux piézométriques tendent à rejoindre l'altitude de la surface de base des thalwegs.

Cette dernière hypothèse présente la surface de base des thalwegs comme la limite inférieure jamais atteinte. En effet, les cours d'eau pérennes que nous allons considérer sont des cours d'eau principaux qui ne se réduisent pas à des petits ruisseaux et ne tarissent pas, même en fin de saison sèche. De plus, le fleuve Sassandra est un cours d'eau à tarissement long, dont la phase finale se déroule en régime influencé par la reprise des pluies de la nouvelle saison humide (SAVANÉ et al., 2003). De façon empirique, WYNS et al. (2004) ont observé une corrélation linéaire entre les paramètres a et b qui représentent respectivement le dénivelé entre la surface du sol et la surface de base des thalwegs et le dénivelé entre la surface piézométrique et la surface de base des thalwegs. Ainsi, connaître en tout point la surface topographique et la surface de base des thalwegs des cours d'eau pérennes serait suffisant pour fournir un niveau piézométrique théorique.

Pour mettre en œuvre cette méthode, il faut disposer d'une carte de distribution spatiale de points cotés sur les cours d'eau pérennes. Pour générer cette carte, 220 points ont été relevés manuellement sur le MNA, avec, comme indiqué plus haut, une erreur verticale absolue de $10 \mathrm{~m}$.

En pointant sur le MNA, à intervalle régulier le long du tracé des cours d'eau pérennes de la zone d'étude, nous avons extrait en chaque point la valeur de $\mathrm{Zr}$ (cote du cours d'eau) représentative d'une maille carrée de $90 \mathrm{~m}$ x $90 \mathrm{~m}$ ce qui donne une valeur $\mathrm{Zr}$ supérieure à celle, réelle, du lit du cours d'eau dans la maille. Cette différence est en théorie d'autant plus importante que le cours d'eau est encaissé ou excentré dans la maille. Les informations relevées pour chaque point sont les coordonnées géographiques (latitude et longitude) et la cote Zr. Elles ont permis d'établir la carte altimétrique de la surface de base des thalwegs des cours d'eau permanents.

Les données de piézométrie utilisées proviennent de la détermination de la profondeur du niveau piézométrique (Npi) des forages. Afin de la traduire en niveau altimétrique, l'altitude du terrain (Zsol) a été utilisée. Ces altitudes proviennent soit des dossiers forages, soit des relevés que nous avons faits lors de nos déplacements sur le terrain ou encore du MNA. On obtient alors l'altitude du niveau piézométrique ponctuel (Zpi).

Pour rechercher des corrélations, nous avons considéré les données des forages où l'altitude de la tête du puits est enregistrée. Ainsi, les relations trouvées ont été généralisées en utilisant, au lieu des notations ponctuelles Zpi et Zsol, les notations $\mathrm{Zp}, \mathrm{Zt}$ et $\mathrm{Zr}$ pour les altitudes respectives de la surface piézométrique, de la surface topographique et de la surface de référence (base des thalwegs) en tout point de la carte modélisée.

\section{RÉSULTATS ET DISCUSSION}

\subsection{Résultats}

La figure 4, obtenue après interpolation par krigeage, présente la surface de base des thalwegs obtenue après

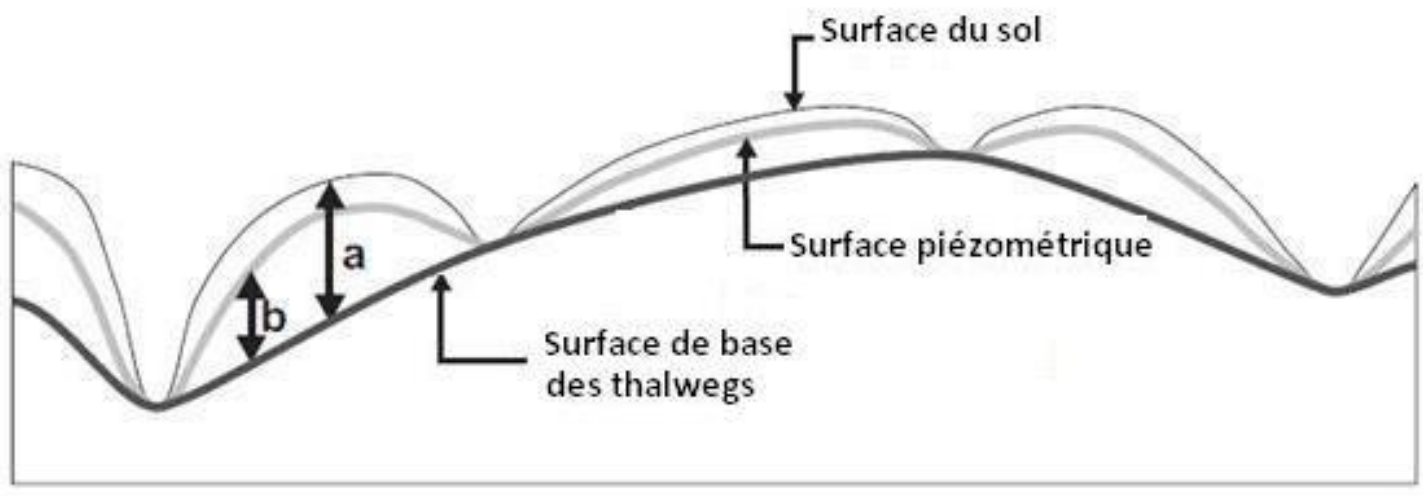

Figure 3. Principe de modélisation de la surface piézométrique (selon WYNS et al., 2004). Principle of water-table modelling (from WYNS et al., 2004). 


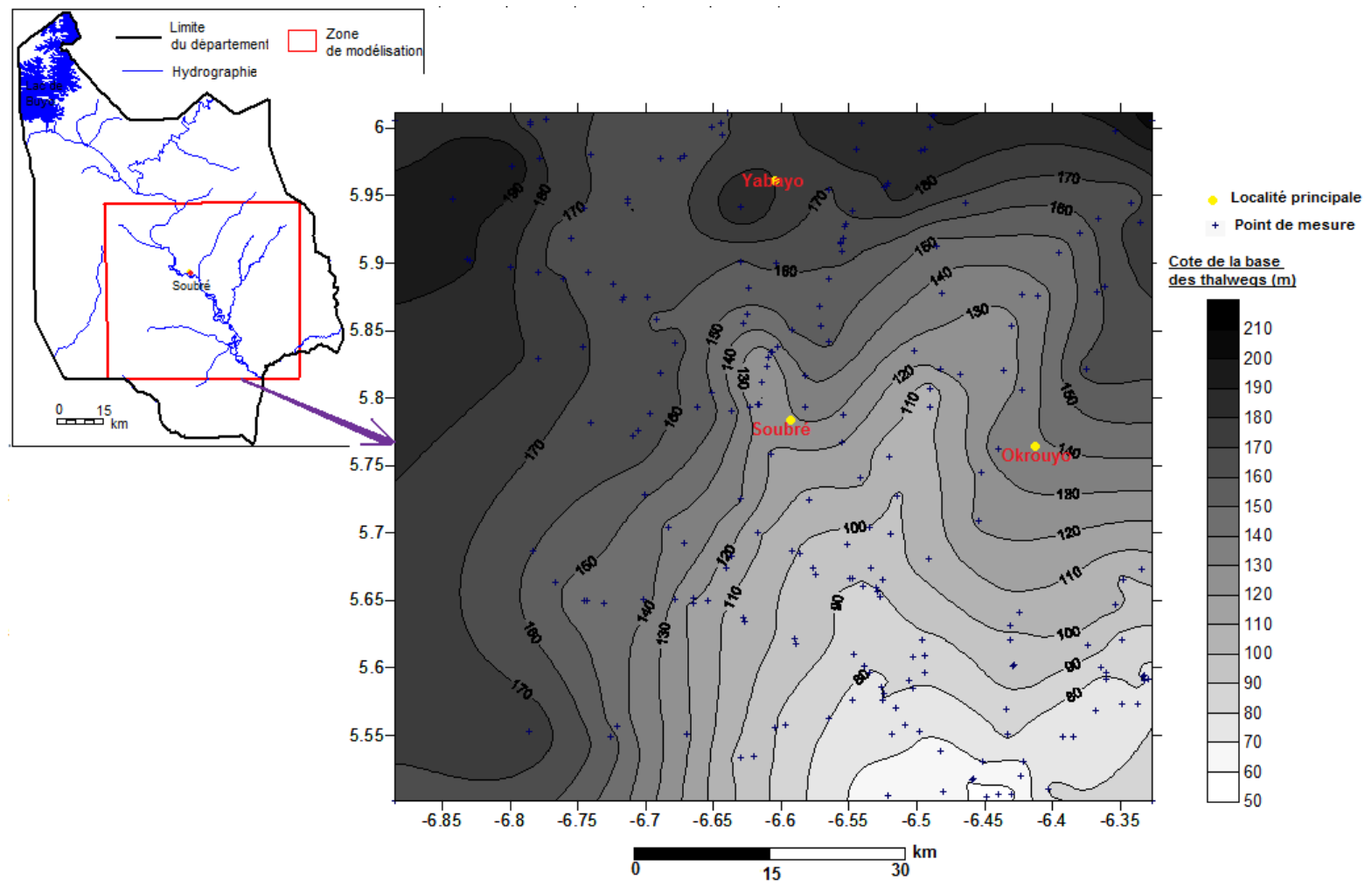

Figure 4. Carte isohypse de la base des thalwegs dans le secteur sud (220 points). Thalweg basal isohypse map in the southern sector (220 points).

modélisation en utilisant les données sur les thalwegs de la figure 5. Cette figure est appelée surface de référence ( $\mathrm{Sr}$ ) et sa résolution, identique à celle du Modèle Numérique d'Altitude, paraît suffisante (Figure 5). Grâce à la faible variance du MNA, il n'y a pas d'anomalie statistique due à la variabilité de la surface et au faible nombre de points.

La figure 5 présente le MNA en isovaleurs et montre la variabilité associée à la surface topographique générale $(\mathrm{Zt})$ de la zone d'étude. Elle rend compte de la géomorphologie générale du secteur étudié où l'on aperçoit la configuration du réseau hydrographique (fond blanc) et les zones d'altitudes relativement importantes en noir. Elle montre aussi la position des forages dont les données piézométriques ont finalement été retenues pour notre modélisation piézométrique.

L'exploitation des informations altimétriques issues des surfaces Sr et St (surface topographique) et des données de forages a permis de calculer les valeurs des paramètres a et $\mathrm{b}$. Le résultat est donné par la figure 6 . Sur ce graphe est ajustée une courbe de tendance linéaire d'équation (1) :

$$
\mathrm{b}=0,9758 \times \mathrm{a}-8,9506
$$

avec $\mathrm{R}^{2}=0,964$

Cette équation présente un coefficient de détermination proche de 1, ce qui implique que la relation est significative, validant ainsi les hypothèses de base émises dans la méthodologie. Néanmoins, nous observons sur le graphe de la figure 6 que neuf sur 61 points présentent des valeurs de b et a négatives.

Ces neufs points qui semblent aberrants au vu des hypothèses et certains forages dont les coordonnées géographiques sont douteuses ont été éliminés. Seuls les forages dont nous avons déterminé ou vérifié les coordonnées géographiques lors de la phase de terrain ont été gardés, réduisant ainsi le nombre à 37 . Le résultat obtenu est satisfaisant (Figure 7) : le coefficient de détermination a été amélioré, soit $\mathrm{R}^{2}=0,97$. Les points sur ce graphique sont moins dispersés que précédemment. Les points qui ont une valeur de a négative (nappe théoriquement artésienne) ne sont plus que 2 sur 37 , soit $5 \%$. La relation s'écrit suivant l'équation ci-dessous :

$$
b=1,0033 \times a-9,6407
$$

En posant que la variable b représente la différence entre la surface piézométrique recherchée (Zpthéo) et la surface de 

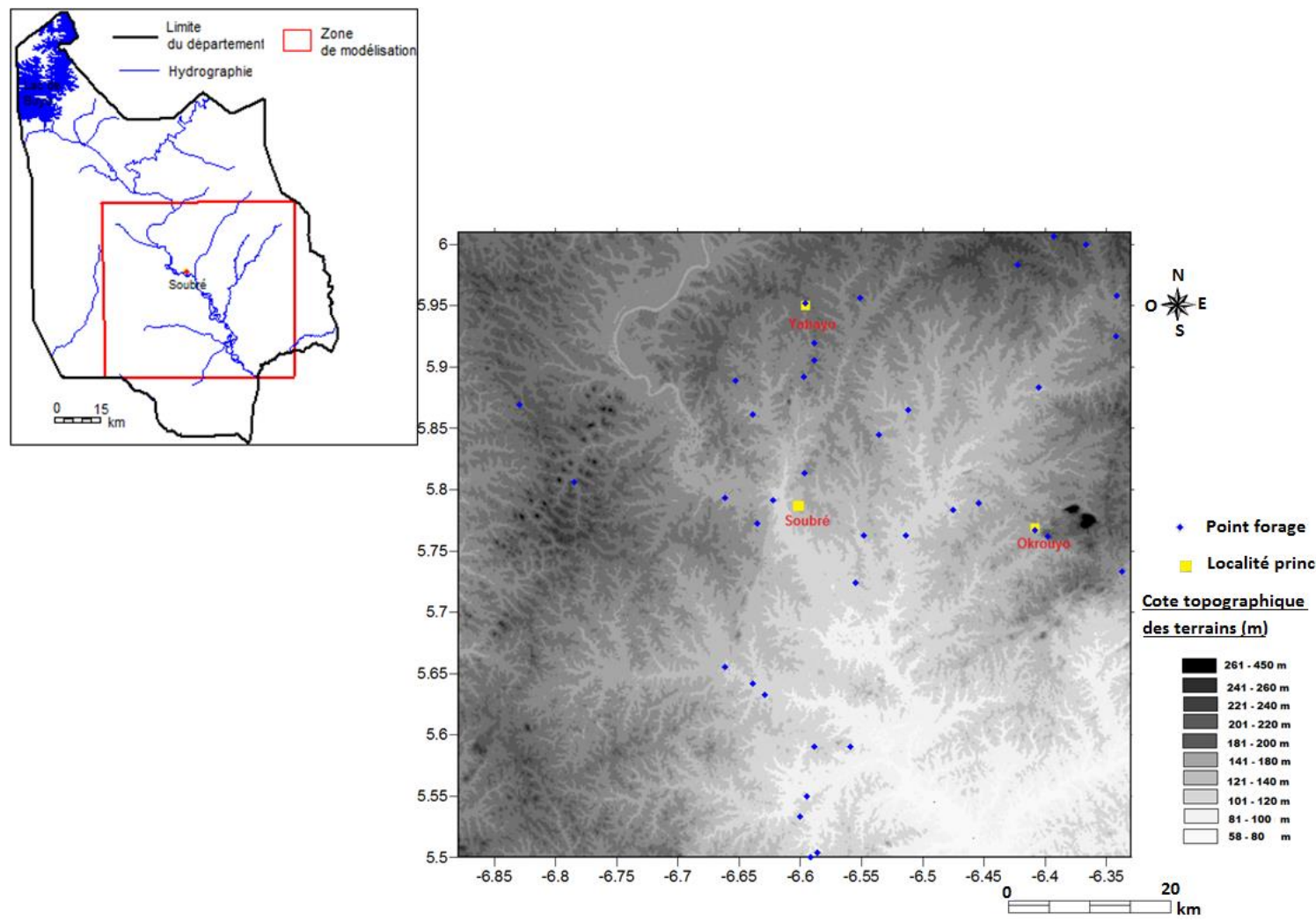

Figure 5. Le modèle numérique d'altitude (MNA) et la localisation des forages utilisés pour la modélisation de la surface piézométrique.

Digital elevation model (DEM) and the location of the drilling used for the modeling of the piezometric surface.

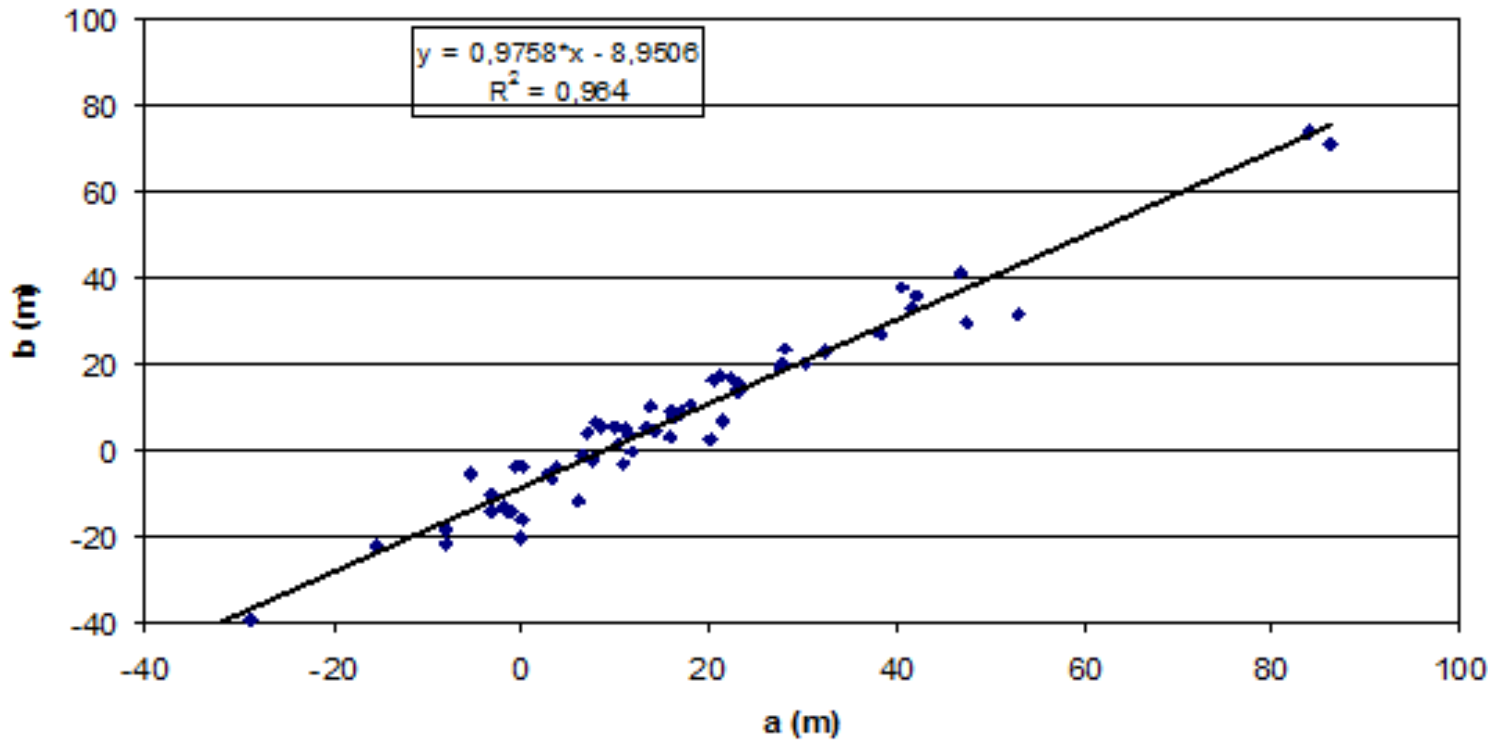

Figure 6. Relation entre la surface piézométrique, l'altitude du sol et l'altitude de la surface de base des cours d'eau pérennes (61 mesures piézométriques réalisées en forages).

Relationship among the water table, the ground elevation and the basal thalweg surface (61 piezometric readings from drilling). 


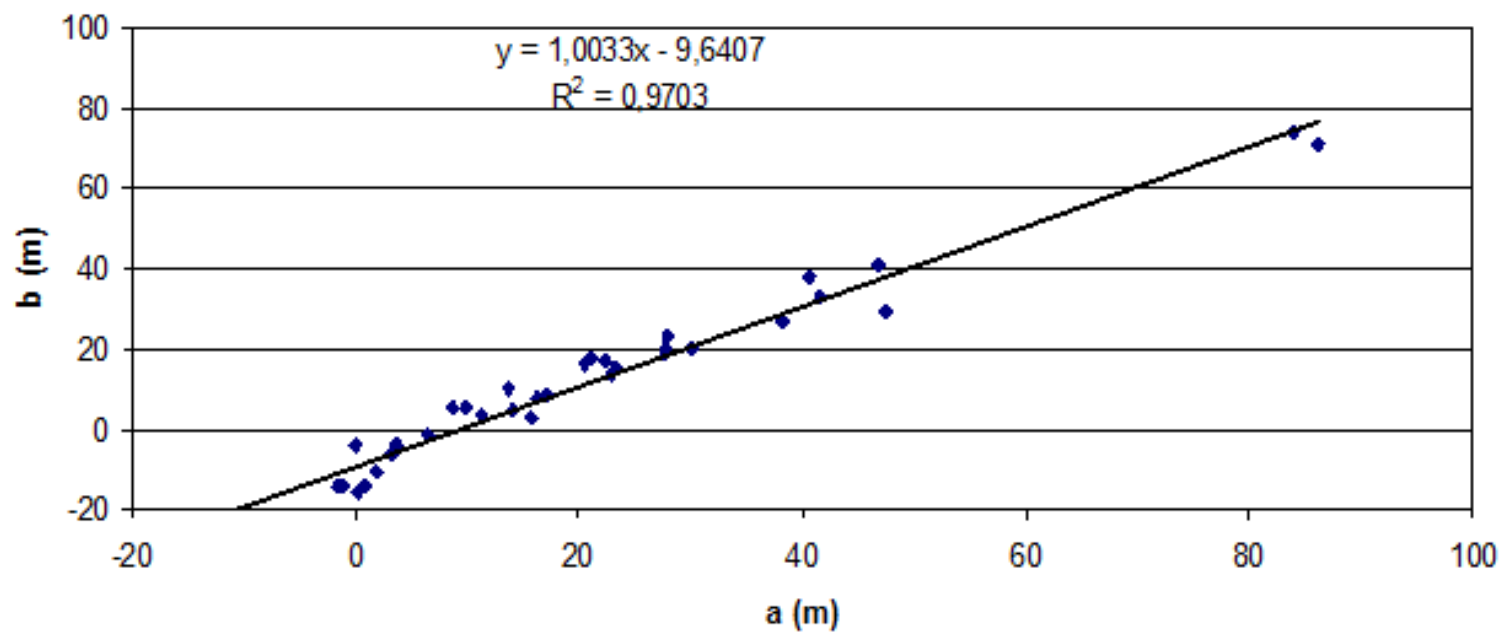

Figure 7. Relation entre la surface piézométrique, l'altitude du sol et l'altitude de la surface de base des cours d'eau pérennes: 37 mesures piézométriques réalisées en forages sélectionnées pour la modélisation de la surface piézométrique. Relationship among the water table, the ground elevation and the basal thalweg surface (37 piezometric readings from drilling)

base des rivières $(\mathrm{Zr})$ et que a est la différence entre $\mathrm{Zt}$ et $\mathrm{Zr}$, et en remplaçant simplement les variables a et $\mathrm{b}$ de l'équation ci-dessus par leurs valeurs, l'équation 2 devient :

$$
\text { Zpthéo = 1,0033 x }(\mathrm{Zt}-\mathrm{Zr})+\mathrm{Zr}-9,6407
$$

Cette équation est utilisée pour établir la carte de la surface piézométrique où la variable $\mathrm{Zt}$ est donnée par le MNA et la variable $\mathrm{Zr}$ par la carte de la figure 4. On obtient la carte de la figure 8 qui représente la surface piézométrique modélisée en première approche.

Cependant, en zone de socle, et particulièrement en milieu plutonique et metamorphique, les cours d'eau permanents représentent les exutoires des eaux souterraines. Aussi, dans le souci de présenter une autre approche de la méthode de WYNS et al. (2004), avons-nous éliminé les forages où le paramètre b est négatif. En outre, nous avons pris en compte des données de forages non visités lors de nos missions de terrain dont les coordonnées géographiques ont été vérifiées et validées à l'aide du MNA. Nous obtenons une base de données comprenant 55 forages et une nouvelle relation d'expression :

$$
\mathrm{b}=0,92 \times \mathrm{a}-7,00 \mathrm{~m}
$$

Avec un coefficient de détermination de $\mathrm{R}^{2}=0,95$ (Figure 9).

Pour créer une possible convergence de la surface piézométrique avec sa surface de référence aux alentours des cours d'eaux pérennes, nous avons considéré les points dont la valeur du paramètre a est inférieure à $60 \mathrm{~m}$. Ainsi, nous obtenons une fonction linéaire d'équation :

$$
\begin{gathered}
\mathrm{b}=0,74 \mathrm{x} \text { a } \\
\text { avec } \mathrm{R}^{2}=0,90
\end{gathered}
$$

Pour obtenir un meilleur coefficient de détermination, une autre solution a été de sélectionner la fonction polynomiale d'expression (Figure 9) :

$$
\begin{gathered}
\mathrm{b}=0,004 \times \mathrm{a}^{2}+0,503 \times \mathrm{a} \\
\text { où } \mathrm{R}^{2}=0,95
\end{gathered}
$$

La relation linéaire ou polynomiale permet de déduire la surface piézométrique en toute maille de $90 \mathrm{~m}$ x $90 \mathrm{~m} \mathrm{du}$ MNA à l'aide d'une transformation simple de la surface de base des thalwegs $(\mathrm{Sr})$ :

$$
\begin{aligned}
& \mathrm{Zp}-\mathrm{Zr}=0,74(\mathrm{Zt}-\mathrm{Zr}) \\
& \mathrm{Zp}=0,74 \mathrm{Zt}+0,26 \mathrm{Zr}
\end{aligned}
$$

Au-delà de la valeur de $\mathrm{a}=60 \mathrm{~m}$, il faudra appliquer la relation affine à pente proche de 1 car, malgré une certaine variabilité de la carte de la profondeur du niveau piézométrique la surface piézométrique au niveau du bassin versant est parallèle à la topographie d'une manière générale.

L'équation 8 est considérée comme une adaptation de l'équation 3 aux conditions locales et à l'insuffisance dans nos données. Ainsi, son exploitation permet d'obtenir une autre carte de présentation de la surface piézométrique modélisée 


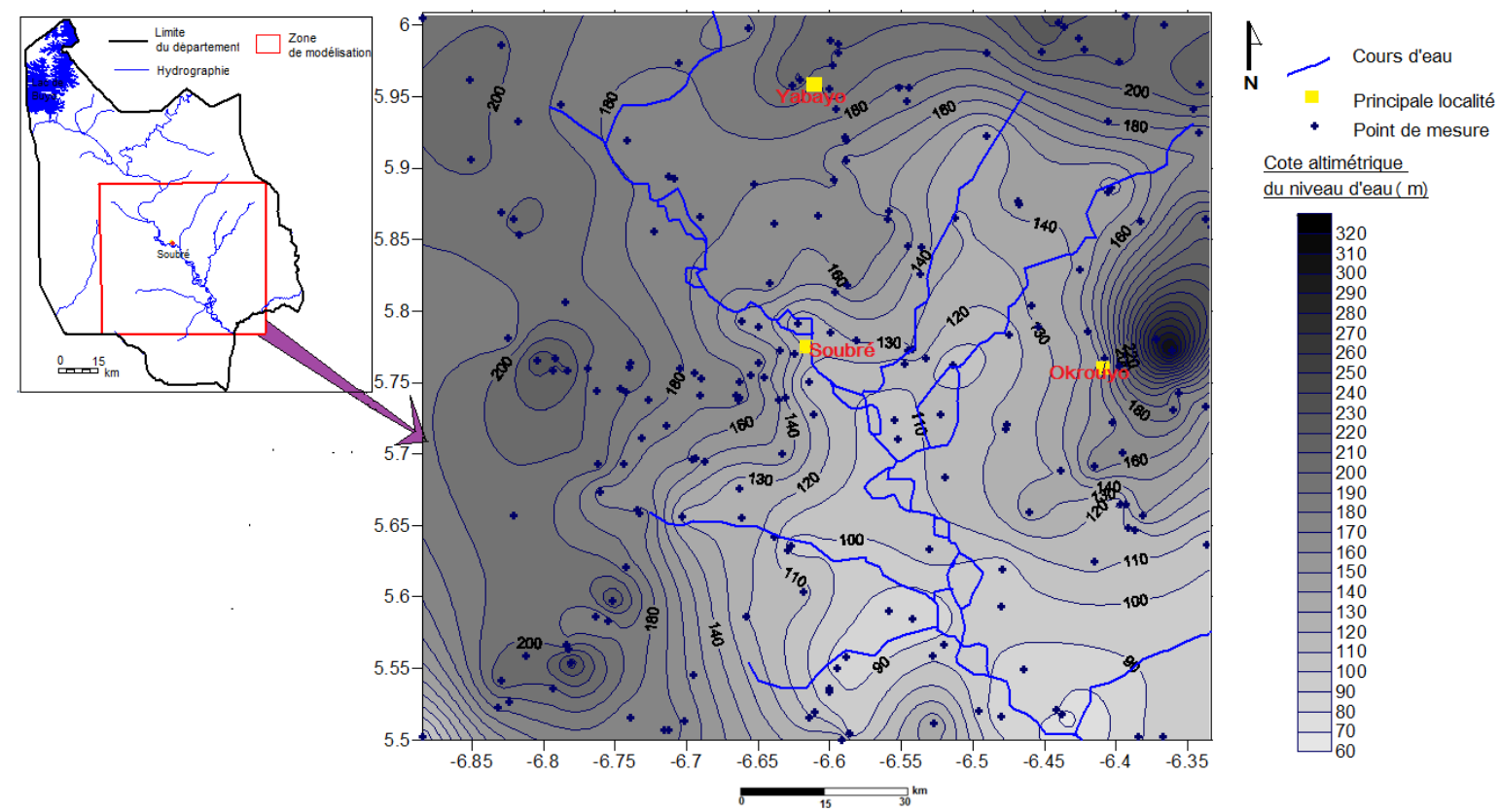

Figure 8. Courbes isovaleurs de la piézométrie théorique dans le secteur d'étude obtenues à l'aide de l'équation $\mathrm{a}=\mathrm{f}(\mathrm{b})$, de la surface de base des thalwegs et de la surface du MNA.

Theoretical piezometric isovalue in the study area obtained by using the equation $a=f(b)$, the basal thalweg surface and the Digital Elevation Model (DEM) surface.

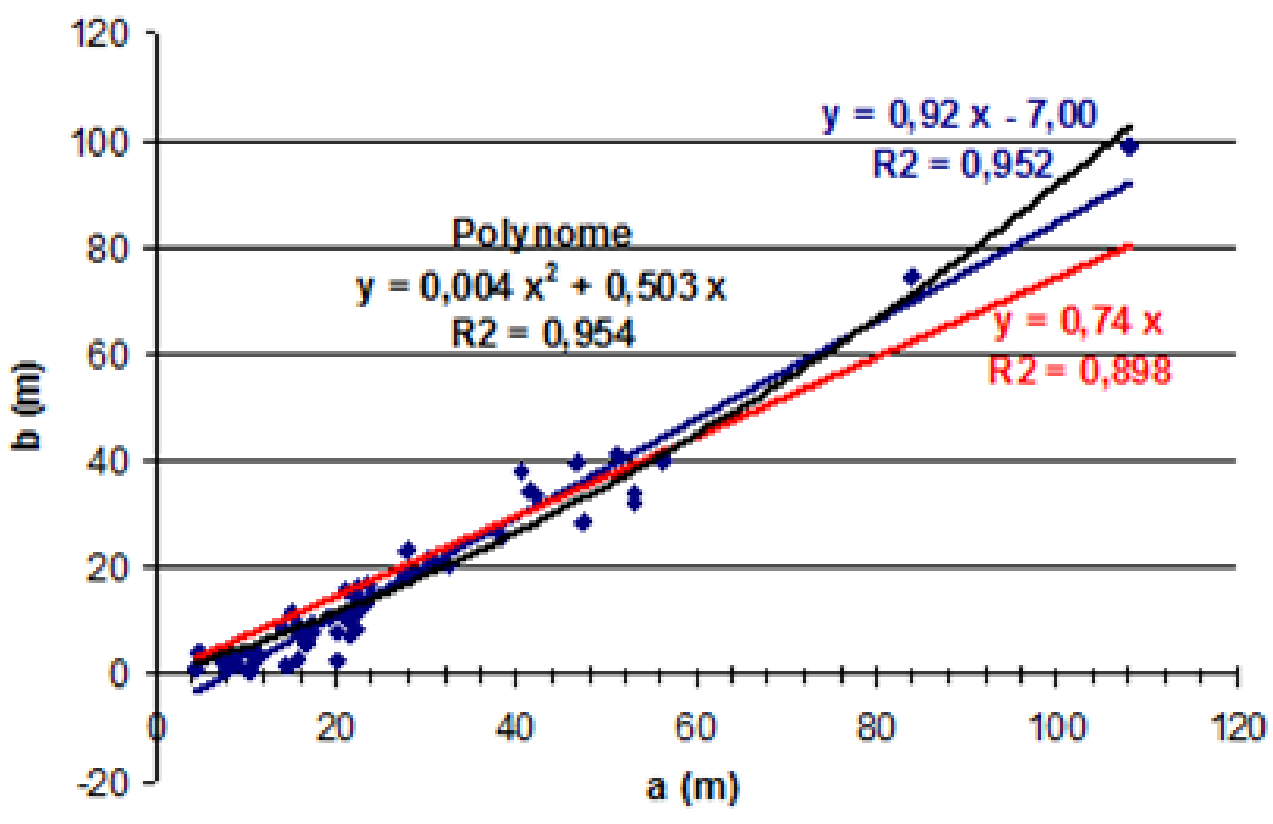

Figure 9. Corrélation de Wyns sur 55 forages, sans les valeurs de b négatives. Wyns correlation from 55 drillings, without the negative values of $b$. 
dans les formations de socle du bassin versant du Sassandra (Figure 10).

\subsection{Discussion}

Pour modéliser la surface piézométrique, nous avons eu recours à un aspect des travaux de WYNS et al. (2004). La détermination d'une carte d'isovaleurs de piézométrie en zone de socle s'avère délicate du fait de la nature discontinue des aquifères dans le socle. Cependant, selon YAO (2012), la zone d'étude est fortement fracturée (présence d'une couverture d'altérites et d'un horizon fissuré bien développé) ; c'est pourquoi nous l'avons considérée comme un milieu continu. La donnée initiale à la modélisation piézométrique est l'établissement de la surface de référence $(\mathrm{Sr})$ à partir des altitudes des points pris sur les cours d'eau pérennes. La pente moyenne le long des principaux cours d'eau et des différentes vallées (sèches ou humides) est inférieure à 1,6\%, valeur inférieure à la pente des terrains de la zone d'étude qui est comprise entre $2 \%$ et $4 \%$ (YAO, 2009). On observe une faible variation des cotes des points relevés pour l'établissement de la surface de référence. La valeur d'altitude extraite est donc supérieure à celle du lit du cours d'eau dans la maille de $90 \mathrm{~m}$ x $90 \mathrm{~m}$ de l'image MNA, avec un excès d'autant plus important que le cours d'eau est encaissé ou excentré dans la maille : cet excès est d'environ $0,5 \mathrm{~m}$ pour une pente de $2 \%$. La précision à ce niveau du MNA ne représente pas une difficulté importante pour le relevé des données. Les 220 points utilisés pour établir Sr s'avèrent suffisants puisque ces points sont assez dispersés pour faire une interpolation. Les surfaces obtenues sont représentatives des phénomènes décrits (base des thalwegs, surface topographique et surface piézométrique). Toutefois, nous savons que certaines altitudes peuvent être exagérées et induire des biais dans la détermination des valeurs des paramètres a et $\mathrm{b}$. C'est pourquoi nous avons reconsidéré les données qui ont permis d'établir l'équation 2. En effet, si l'on suppose que la surface de base des thalwegs est représentative de la réalité, des valeurs de a et b négatives ne sont pas correctes. Cela signifierait que soit :

- $\quad b<0$ : le niveau d'eau dans certains forages est situé en dessous de la surface de base des thalwegs, ce qui peut s'expliquer si l'on a considéré des vallées sèches comme des vallées pérennes au moment d'élaborer la surface de base qui serait donc trop haute. Cette valeur négative de $b$ s'explique le plus souvent par le rabattement de la nappe dû au pompage de l'eau dans le forage ou à son voisinage ;

- $\quad \mathrm{a}<0$ : la tête de certains forages est située en dessous de la surface de base des thalwegs; ce second cas parait illogique à priori, mais est possible si le forage se trouve dans une dépression non prise en compte lors de la modélisation de la surface de base des thalwegs, qui serait donc là aussi trop haute ou si, localement, le forage est artésien.

L'inexactitude des coordonnées de certains forages peut aussi expliquer ces valeurs négatives. Dans WYNS et al. (2004), la valeur négative de l'ordonnée b à l'origine est due à l'encaissement moyen de la surface des cours d'eau par rapport

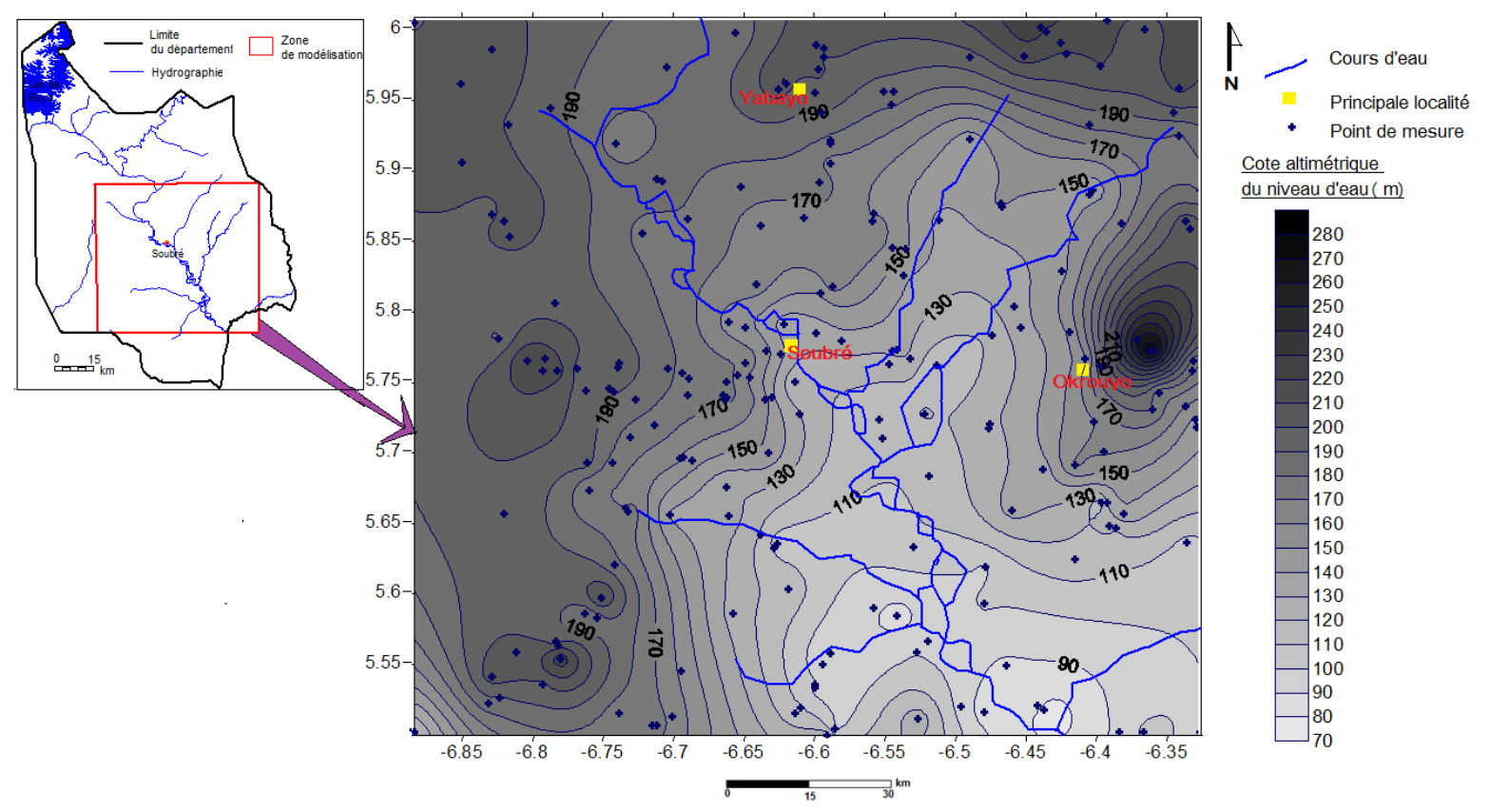

Figure 10. Courbes isovaleurs de la piézométrie théorique d'après la corrélation de Wyns modifiée. Theoretical piezometric isovalues obtained by using modified Wyns correlation. 
à leurs berges, le MNA donnant la cote du sol et non de la surface de l'eau.

Les travaux de WYNS et al. (2004) ont été pour nous une base scientifique pour aboutir à l'expression des équations 2 et 3. La pente voisine de l'unité de la droite de régression des équations 2 et 4 indique un milieu de faible perméabilité à l'échelle du massif, ce qui est bien le cas en contexte de socle : en effet, lorsque la perméabilité tend vers l'infini la pente tend vers 0 (la surface piézométrique et la surface des rivières pérennes sont confondues), tandis que lorsque la perméabilité tend vers 0 , la pente tend vers 1 , la surface piézométrique et la surface topographique sont confondues (WYNS et al., 2004). En prenant en compte ces hypothèses et au regard du contexte d'étude, nous avons abouti à l'équation 8 .

L'exploitation des niveaux piézométriques dans les forages, des informations sur la base des thalwegs et des équations 3 et 8 ont permis d'établir les cartes piézométriques théoriques des figures 8 et 10. La figure 8 représente la surface piézométrique issue de l'application directe de la méthode de WYNS et al. (2004). En tenant compte des données de forages, de la géomorphologie et du contexte géologique (roches plutoniques et métamorphiques) de la région de Soubré, nous aboutissons à l'équation 8. Elle permet de construire une nouvelle carte de la surface piézométrique, la figure 10, qui a les mêmes caractéristiques que celle de la figure 8. Cependant, elle montre des surfaces piézométriques moins profondes dans la région d'Okrouyo. De surcroît, elle se rapproche davantage de la carte isohypse de la base des thalwegs, confirmant ainsi que les eaux souterraines de la région circulent en direction des cours d'eau principaux notamment vers le fleuve Sassandra. C'est ce qui justifie que ces cours d'eau soient pérennes même en saison non pluvieuse entre les mois de décembre et de mars (YAO, 2009).

En somme, notons que les eaux souterraines de la région étudiée dans le département de Soubré s'écoulent en conformité avec l'écoulement superficiel. Ceci est logique compte tenu de la perméabilité moyenne à faible des milieux de socle et de la relative homogénéité de leurs propriétés hydrodynamiques à cette échelle d'approche. À même échelle d'étude, le bassin versant hydrogéologique du fleuve Sassandra s'inscrit dans les mêmes limites que le bassin hydrologique. Ce constat démontre que l'équation 8 permet de modéliser la surface piézométrique dans un contexte de socle précambrien. Certes, ce résultat découle de la méthode de WYNS et al. (2004), mais il présente une nouvelle expression qui montre nettement le lien entre la cote topographique et la base des thalwegs. Cette expression pourrait être affinée en enrichissant la base de données initiale, notamment la manière de relever les points pour l'établissement de la carte de base des thalwegs (Figure 4). Un relevé automatisé donnera plus de points qui pourront améliorer la précision de la carte de la base des thalwegs qui est un paramètre primordial dans la modélisation piézométrique.
Bien avant, notons que les données récoltées manuellement rendent compte de la réalité du terrain. En effet, la valeur théorique moyenne de la profondeur de l'eau souterraine calculée en se référant à l'équation 8 est de $7,1 \mathrm{~m}$. Cette valeur théorique du niveau de l'eau est similaire à celle observée sur le terrain. En effet, les travaux de YAO (2009) ont montré que la profondeur moyenne du niveau piézométrique $(\mathrm{Np})$ est de 7,3 m dans le département de Soubré.

Par la méthodologie utilisée et en exploitant les données des niveaux piézométriques des forages et l'équation 8 , la carte piézométrique théorique nouvelle (Figure 10) a été établie. Ainsi, à partir de la figure 10 ou de l'équation 8 , nous pouvons déterminer le niveau piézométrique en tout point situé dans la zone de la modélisation. Dans la Figure 10, la nappe n'atteint pas une altitude maximale si importante que dans la figure 8 , mais elle apparait en revanche un peu plus haute en certains points situés à proximité des confluences (plus haute de $10 \mathrm{~m}$ environ).

Toutefois, la validation du modèle mathématique exprimé par l'équation 8 a permis de mettre en évidence des écarts entre le niveau piézométrique mesuré en forage et cette surface théorique. Ces anomalies pourront s'interpréter selon une grille de lecture que nous ébauchons ici.

Une anomalie haute du niveau piézométrique peut signifier que l'aquifère est alimenté localement par les eaux du cours d'eau. Cela semble peu probable à l'échelle de travail mise en œuvre et est très vraisemblablement dû aux imprécisions du MNA ou de la méthode (YAO, 2009 ; YAO et al., 2010, 2012). Une anomalie basse du niveau piézométrique peut se rencontrer là où les eaux souterraines sont rabattues par un drainage naturel (zone de fractures) ou artificiel (pompage).

\section{CONCLUSION}

Au terme de cette étude, on note que dans le bassin versant du Sassandra, la surface piézométrique est corrélée à la surface topographique. Malgré une certaine variabilité de la carte de la profondeur du niveau piézométrique, le niveau moyen d'eau dans les forages varie entre 7,1 et 7,3 m. Le niveau piézométrique dans la nappe a été modélisé pour compenser les insuffisances dans les données de la piézométrie. Nous pouvons à travers l'équation $\mathrm{Zp}=0,74 \mathrm{Zt}+0,26 \mathrm{Zr}$ calculer directement le niveau piézométrique en tout point en connaissant les coordonnées géographiques et la cote topographique. En outre, on déduit que le bassin versant hydrogéologique se superpose au bassin hydrologique. Ces résultats constituent une première étape dans l'étude hydrologique du bassin versant du Sassandra. 
En perspective, ces résultats pourront être vérifiés ou affinés avec l'utilisation d'une image MNA de grande résolution, une acquisition automatisée pour la densification des points ce qui permettra de spatialiser la relation obtenue en fonction de la lithologie (schiste, roches cristallines et volcano-sédimentaire). Un suivi à plus long terme précisera l'importance de l'infiltration et des écoulements de subsurface. In fine, nos travaux aideront à la gestion intégrée des ressources en eaux (souterraines et de surface) de la région face à la vulnérabilité de ces aquifères par rapport aux pesticides qui sont utilisés dans les nombreuses plantations de cultures de rente (cacao, café, palmier à huile et hévéa).

\section{REMERCIEMENTS}

Les auteurs tiennent à remercier la Sous-direction Territoriale de l'Hydraulique de San Pedro pour les données mises à leur disposition. Nos remerciements vont aussi à l'endroit des villageois pour leur disponibilité et participation active à l'acquisition des données de terrain.

\section{RÉFÉRENCES BIBLIOGRAPHIQUES}

ARDOUIN, B.S. (2004). Variabilité hydroclimatique et impacts sur les ressources en eau des grands bassins hydrographiques en zone soudano-sahélienne. Thèse de Doctorat, Univ. Montpellier II, France, 437 p. + Annexes.

BELIARD, C., F. LELONG et PELISSIER (1973). Premiers résultats de l'étude des nappes phréatiques contenues dans les formations d'altération. Série doc. 8, Univ. Abidjan, Côte d'Ivoire.

BIÉMI, J. (1992). Contribution à l'étude géologique hydrogéologique et par télédétection des bassins versants subsahariens du socle précambrien d'Afrique de l'Ouest : hydrostructurale, hydrodynamique, hydrochimie et isotopique des aquiferes discontinus de sillons et aires granitiques de la haut Marahoué (Côte d'Ivoire). Thèse de Doctorat d'État, Univ. Abidjan, Côte d'Ivoire, 480 p.

BRGM-CIEH (1972). Étude du ruissellement et de l'infiltration sur un bassin versant de zone de savane (Korhogo-Côte d'Ivoire). CIEH, série Hydrogéologie, 67 p.

DEWANDEL, B., P. LACHASSAGNE, R. WYNS, J.C MARECHAL et N.S. KRISHNAMURTHY (2006). A generalized 3-D geological and hydrogeological conceptual model of granite aquifers controlled by single or multiphase weathering. J. Hydrol., 330, 260-284.

DEWANDEL, B., P. LACHASSAGNE, F.K. ZAIDI, S. CHANDRA (2011). A conceptual hydrodynamic model of a geological discontinuity in hard rock aquifers: Example of a quartz reef in granitic terrain in South India. J. Hydrol., 405, 474-487.

ENGALENC, M. (1978). Méthode d'étude et de recherche de l'eau souterraine des roches cristallines de l'Afrique de l'Ouest. CIEH. Maisons-Alfort, France, 318 p.

FAILLAT, J.P. et P. LEBLOND (1982). Premiers résultats des essais menés sur une station de pompage expérimentale en Côte d'Ivoire. Coll. Milieux discontinus en Hydrogeol. BRGM (45), 301-314.

FAILLAT, J-P. et B. BLAVOUX (1989). Caractères hydrochimiques des nappes des roches endogènes en zone tropicale humide : l'exemple de la Côte d'Ivoire. J. Afr. Earth Sci., 9, 31-40.

JOSEPH, C. (1969). Interprétation des mesures disponibles des variations naturelles du niveau des nappes en Côte d'Ivoire. CIEH, 58 p.

KAMAGATE, B. (2006). Fonctionnement hydrologique et origine des écoulements sur un bassin versant en milieu tropical de socle au Benin : bassin versant de la Donga (haute vallée de l'Ouémé). Thèse de doctorat. Univ. Montpellier 2, France, 319 p.

LACHASSAGNE, P., R. WYNS, B. DEWANDEL (2011). The fracture permeability of hard rock aquifers is due neither to tectonics, nor to unloading, but to weathering processes. Terra Nova, 23, 145-161

LAROCQUE, M. et M.C. PHARAND (2010). Dynamique de l'écoulement souterrain et vulnérabilité d'un aquifère du piémont appalachien (Québec, Canada). Rev. Sci. Eau, 23, 73-88.

LELONG, F. et J. LEMOINE (1968). Les nappes phréatiques des arènes et des altérations argileuses. Leur importance en zone intertropicale; les difficultés de leur exploitation. Bull. $B R G M$ série III, 41-52.

LENCK, P.P. (1977). Données nouvelles sur l'hydrogéologie des régions à substratum métamorphique ou éruptif. Enseignements tirés de la réalisation de 900 forages en Côte d'Ivoire. C.R. Acad. Sc. Paris, 28, 497-500. 
MinistèrequébécoisdesRessources naturelles, delaFaune(2011). Caractéristiques géométriques des images issues des modèles numériques d'attitudes. http://www.mrnf.gouv.qc.ca/ publications/territoire/expertise/13_MNA. (consultation le 20 février 2011).

PAPON, A. et R. LEMARCHAND (1973). Géologie et minéralisation du sud-ouest de la Côte d'Ivoire. Synthèse des travaux de l'opération SASCA (1962-1968). SODEMI, Abidjan, $284 \mathrm{p}$.

SAVANE, I., K.M. COULIBALY et GIOAN, P. (2003). Étude comparative de trois méthodes de calcul du coefficient de tarissement des cours d'eau. Science et changements planétaires. Sécheresse, 14, 37-42.

TAGINI, B. (1971). Esquisse Structurale de la Côte d'Ivoire. Essai de géotechnique régionale. Thèse d'État, Univ. Lausanne, Suisse, $302 \mathrm{p}$.

WYNS, R., J.C. GOURRY, J.M. BALTASSAT et F. LEBERT (1999). Caractérisation multiparamètres des horizons de subsurface $(0-100 \mathrm{~m})$ en contexte de socle altéré, in : 2e Colloque GEOFCAN, BRGM, IRD, UPMC, pp. 105110.

WYNS, R., J.M. BALTASSAT, P. LACHASSAGNE, A. LEGCHENKO, J. VAIRON et F. MATHIEU F. (2004). Application of proton magnetic resonance sounding to groundwater reserve mapping in weathered basement rocks (Brittany France). Bull. Soc. Géol. Fr., 1, 21-34.

YAO, K.T. (2009) Hydrodynamisme de l'eau souterraine dans les aquiferes de socle cristallin et cristallophyllien du Sud-Ouest de la Côte d'Ivoire : cas du département de Soubré. Apports de la télédétection, de la géomorphologie et de l'hydrogéochimie. Thèse de doct. Conservatoire national des arts et métiers, Paris, France, 284 p.

YAO, K.T., O. FOUCHÉ, M.S. OGA, J. BIÉMI, C. PERNELLE (2010). Circuit de l'eau dans un aquifere de socle hétérogène et fracturé : une enquête hydrochimique dans le bassin versant du Sassandra (Sud-Ouest de la Côte d'Ivoire, 5e conférence African Association of Women in Geosciences (CAAWG5) «Femmes et Géosciences pour la Paix», 11-17 Avril 2010, Grand Bassam, Côte d'Ivoire.

YAO, K.T., O. FOUCHÉ, Y. M.S. OGA et T. ASSOMA (2012). Extraction de linéaments structuraux à partir d'images satellitaires, et estimation des biais induits, en milieu de socle précambrien métamorphisé. Rev. Télédétection, 10, 161-178. 